\title{
Study of the Helium Enrichment in Globular Clusters
}

\author{
Aldo A. R. Valcarce \& Márcio Catelan \\ Pontificia Universidad Católica de Chile, \\ Departamento de Astronomía y Astrofísica, \\ Av. Vicuña Mackenna 4860, 782-0436 Macul, Santiago, Chile \\ email: avalcarc@astro.puc.cl; mcatelan@astro.puc.cl
}

\begin{abstract}
Globular clusters (GCs) are spheroidal concentrations typically containing of the order of $10^{5}$ to $10^{6}$, predominantly old, stars. Historically, they have been considered as the closest counterparts of the idealized concept of "simple stellar populations." However, some recent observations suggest than, at least in some GCs, some stars are present that have been formed with material processed by a previous generation of stars. In this sense, it has also been suggested that such material might be enriched in helium, and that blue horizontal branch stars in some GCs should accordingly be the natural progeny of such helium-enhanced stars. In this contribution we show that, at least in the case of M3 (NGC 5272), the suggested level of helium enrichment is not supported by the available, high-precision observations.
\end{abstract}

Keywords. Hertzsprung-Russell diagram, stars: abundances, stars: evolution, stars: horizontalbranch, globular clusters: general, globular clusters: individual (M3 = NGC 5272)

\section{Introduction}

Globular clusters (GCs) have long been thought to provide one of the closest approximations to the idealized concept of "simple stellar populations," with all stars in a given GC having closely the same distance, age, and chemical composition. However, our understanding of these objects is quickly changing, thanks not only to measurements of chemical inhomogeneities, but also to improved color-magnitud diagrams (CMDs), which show that at least some GCs present multiple populations, presumably indicative of multiple formation episodes.

Besides the classical cases of $\omega$ Cen (e.g., Villanova et al. 2007) and M54 (e.g., Siegel et al. 2007), which host multiple red giant branches (RGBs), subgiant branches (SGBs), and main sequences (MSs), a triple MS has recently been identified in NGC 2808 (Piotto et al. 2007), whereas a double SGB has also been found in 47 Tuc (Anderson et al. 2009), M22 (Marino et al. 2009), NGC 1851 (Milone et al. 2008), NGC 6388 (Piotto 2008), and NGC 5286 (Piotto et al. 2009). Several of these CMD peculiarities often go hand-in-hand with multimodalities along the horizontal branch (HB).

As a rule, different formation episodes inside the clusters have been suggested as the explanation for this phenomenon. However, depending on the specific case considered, different chemical enrichment scenarios have been advocated, including a spread in the CNO elements (Cassisi et al. 2008, Pietrinferni et al. 2009) or/and various populations with different initial He abundances. As to the latter, a helium abundance of $40 \%$ by mass has been found to be required in order to reproduce the bluest MSs in $\omega$ Cen and NGC 2808 (Piotto et al. 2005, 2007).

In addition to this, it has also been recently suggested that the spread in colors seen along the HB in GCs should be due to a spread in $Y$ within these objects, in which case helium abundance enhancements would be the rule, rather than the exception, among GCs (e.g., Caloi \& D'Antona 2007, D'Antona \& Caloi 2008). In this sense, these authors 
have found that the helium abundance among M3's blue HB stars should be enhanced, with respect to M3's red HB population, by at least $\Delta Y=0.02$. The main purpose of this contribution is to test this key prediction (see also Catelan et al. 2009 for more details).

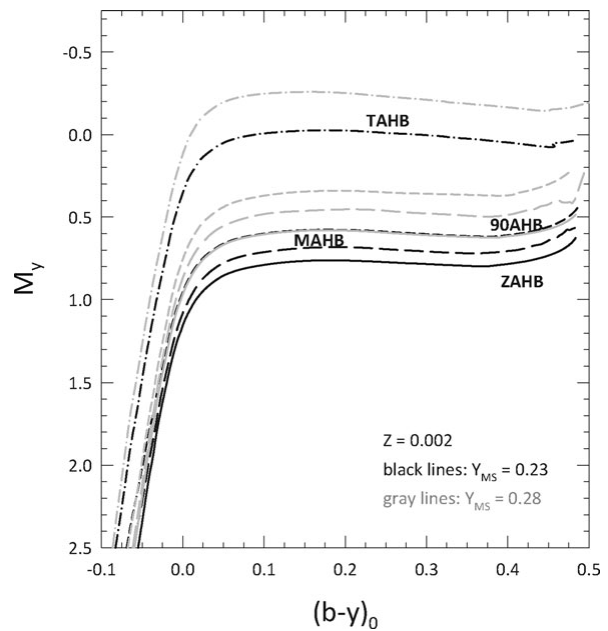

Figure 1. Comparison between the equivalent evolutionary sequences (EESs) for a fixed metallicity and two different helium abundances (black lines: $Y=0.23$; gray lines: $Y=0.28$ ).

\section{Is there a Helium-Enhanced Population in M3?}

To answer this question, we compared observational data for stars in the HB phase with zero age HB (ZAHB) models for different helium abundances.

\subsection{Observational Data and Theoretical Models}

Very briefly, we used the high-precision Strömgren photometry for the cluster, obtained with the Nordic Optical Telescope (Grundahl et al. 1998, 1999).

Theoretical models are from Catelan et al. (1998) and Sweigart \& Catelan (1998), with heavy-element abundances $Z=0.0005,0.001$, and 0.002 , and He abundances $Y=0.23$, 0.28 , and 0.33 . This nicely brackets the possible metallicity range for M3, which in the Zinn \& West (1984) scale and in the Carreta \& Gratton (1997) scale have $[\mathrm{Fe} / \mathrm{H}]=-1.57$ and $[\mathrm{Fe} / \mathrm{H}]=-1.34$, respectively. Taking into account an $[\alpha / \mathrm{Fe}]=+0.27$ for the cluster (Carney 1996), these values imply a $Z=8.3 \times 10^{-3}$ and $Z=1.4 \times 10^{-3}$, respectively, based on the Salaris et al. (1993) $Z-[\mathrm{Fe} / \mathrm{H}]-[\alpha / \mathrm{Fe}]$ relation. Model sequences from VandenBerg et al. (2006) for a $Y=0.236$ and $Z=8.5 \times 10^{-4}$ have also been used, for comparison.

To transfer the evolutionary tracks to the observational plane, we used the color transformations and bolometric correction tables from Clem et al. (2004).

\subsection{Signatures of Varying He Abundances}

To determine how different the HB morphology may become when the He abundance is changed, we used the following equivalent evolutionary sequences (EESs) for this phase: i) The ZAHB; ii) The terminal-age HB (TAHB), or He exhaustion locus; iii) The middleage HB (MAHB); iv) The 90\%-age HB (90AHB). The latter two are the position corresponding to $\mathrm{HB}$ stars that have completed $50 \%$ and $90 \%$ of their total evolutionary history along the HB, respectively. Furthermore, the MAHB could be also assumed as the average position occupied by all HB stars, if there is a continuous star supply from the RGB. 

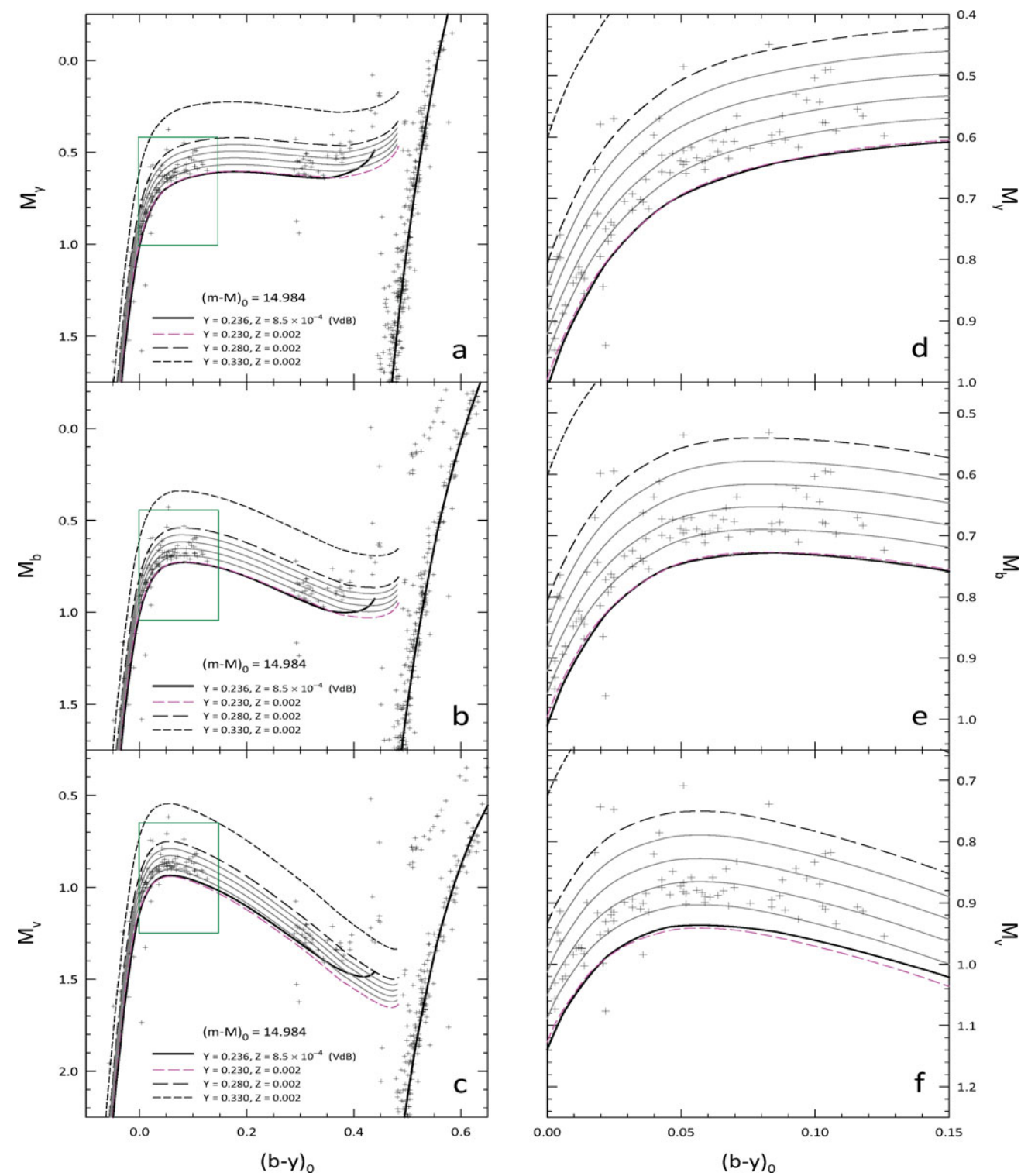

Figure 2. Comparison between the empirical data for M3 and theoretical ZAHBs for the several different $Y$ values indicated. The thin solid lines indicate interpolated ZAHB loci for $Y$ values between 0.24 and 0.27 , in intervals of 0.01 . Panels $d$ through $f$ present expanded views of the rectangular box around the blue HB shown in panels a through c, respectively.

Figure 1 shows how these EESs change when $Y$ changes. Clearly, the luminosity of each EES increases very substantially for larger $Y$ values. Therefore, differences in the He abundance between red and blue HB stars in a given cluster (e.g., Caloi \& D'Antona 2007, D'Antona \& Caloi 2008) should imply a difference in magnitude between red and blue HB stars.

\subsection{Results}

We compare the empirical M3 CMD with ZAHBs for different He abundaces in Figure 2. To produce these plots, we corrected the CMD data for reddening using a $E(B-V)=0.01$ 
(Harris 1996); extinction coefficients for the Strömgren system were taken from Catelan \& Cortés (2008). In these plots, the red HB of the cluster was fit to the ZAHB locus for a canonical $Y=0.23$.

Clearly, when the red HB of the cluster is forced to match ZAHB models with the canonical He abundance, the blue HB stars of the cluster are found to also agree with the same ZAHB locus, without the need for He enhancement. More specifically, our comparison shows that an increase in $Y$ by more than 0.01 among the blue HB stars (with respect to the red HB stars in the same cluster) would not be compatible with the data. This strongly suggests that the level of He enhancement is most likely less than 0.01 among the cool blue HB stars in M3.

In the future, we will apply similar tests to other GCs which have been suggested to harbor He-enhanced populations, thus providing important constraints on the Heenhancement scenario.

\section{Acknowledgement}

We thank F. Grundhal, A.V. Sweigart, and C. Cortés for helpful discussions. Support for A.V. is provided by IAU, CONICYT, SOCHIAS, MECESUP, and ALMA. Support for M.C. is provided by Proyecto Basal PFB-06/2007, by FONDAP Centro de Astrofísica 15010003, by Proyecto FONDECYT Regular \#1071002, and by a John Simon Guggenheim Memorial Foundation Fellowship.

\section{References}

Anderson, J., Piotto, G., King, I. R., Bedin, L. R., \& Guhathakurta, P. 2007, ApJ, 697, L58

Caloi, V. \& D'Antona, F. 2007, A\&广A, 463, 949

Carney, B. W. 1996, PASP, 108, 900

Carreta, E. \& Gratton, R. 1997, A\& AS, 121, 95

Cassisi, S., Salaris, M., Pietrinferni, A., Piotto, G., Milone, A. P., Bedin, L. R., \& Anderson, J. 2008 ApJ, 672, L115

Catelan, M., Borissova, J., Sweigart, A. V., \& Spassova, N. 1998, ApJ, 494, 265

Catelan, M. \& Cortés, C. 2008, ApJ, 676, L135

Catelan, M., Grundahl, F., Sweigart, A. V., Valcarce, A. A. R., \& Cortés, C. 2009, ApJ, 695, L97

Clem, J. L., VandenBerg, D. A., Grundahl, F., \& Bell, R. A. 2004, AJ, 127, 1227

D'Antona, F. \& Caloi, V. 2008, MNRAS, 390, 693

Grundahl, F., Catelan, M., Landsman, W. B., Stetson, P. B., \& Andersen, M. I. 1999, ApJ, 524, 242

Grundahl, F., VandenBerg, D. A., \& Andersen, M. I. 1998, ApJ, 500, L179

Harris, W. E. 1996, AJ, 112, 1487

Marino, A. F., Milone, A. P., Piotto, G., Villanova, S., Bedin, L. R., Bellini, A., \& Renzini, A. 2009, arXiv:0905.4058

Milone, M. H., et al. 2008, ApJ, 673, 241

Pietrinferni, A., Cassisi, S., Salaris, M., Percival, S., \& Ferguson, J. W. 2009, ApJ, 697, 275

Piotto, G., et al. 2005, ApJ, 621, 777

Piotto, G., et al. 2007, ApJ, 661, L53

Piotto, G. 2008, MmSAI, 79, 334

Piotto, G., et al. 2009, in prep.

Salaris, M., Chieffi, A., \& Straniero, O. 1993 ApJ, 414, 580

Siegel, M. H., et al. 2007, ApJ, 667, L57

Sweigart, A. V. \& Catelan, M. 1998 ApJ, 501, L63

VandenBerg, D. A., Bergbusch, P. A., \& Dowler, P. D. 2006, ApJS, 162, 375

Villanova, S., et al. 2007, ApJ, 663, 296

Zinn, R. \& West, M. J. 1984, ApJS, 55, 45 